\title{
Interpreting eGFR
}

\author{
Muzafar Maqsood Wani, D.M., Imtiaz Ahmed Wani, D.M. \\ Department of Nephrology, Sher-i-Kashmir Institute of Medical Sciences, Soura, Srinagar
}

Clinical assessment of kidney function is essential for assessing overall health, interpreting signs and symptoms, selecting the correct dosage for renally excreted drugs, preparing for invasive diagnostic or therapeutic procedures, and detecting/ evaluating/ monitoring acute and chronic kidney diseases. The glomerular filtration rate (GFR) provides a window on renal excretory function. Various ways of estimating or measuring GFR are summarized in box.

\section{Methods to estimate GFR}

- Serum creatinine

- Creatinine clearance (24 hour urine collection with blood samples)

- Calculated/estimated GFR (using CG or MDRD or CKDEPI equation)

- Measured GFR (inulin or EDTA clearance)

Serum creatinine has long been used as a surrogate marker of GFR but it has its own limitations, while as creatinine clearance is bothersome most of the times. ${ }^{1}$ Precise measurement of GFR by inulin clearance or chromium labelled EDTA clearance is not practical in day to day management of patients.

GFR is most commonly estimated (eGFR) rather than measured using formulas which began about 5 decades back. $^{1,2}$ These equations use combination of various patient related indices like serum creatinine level, age, race, sex, body size and ethnicity. These equations continue to be refined and

\section{Correspondence:}

Muzafar Maqsood Wani

Assistant Professor,

Department of Nephrology, Sher-i-Kashmir Institute of Medical

Sciences, Soura, Srinagar, J\&K.

E-mail:mmmwani@gmail.com many laboratories are now reporting the GFR automatically. Since eGFR can often be calculated using data available in most electronic medical record systems, it can be reported directly with any laboratory report that includes a serum creatinine value. Hence, it is imperative that we understand how to interpret the eGFR value and how to recognize when the estimate may not be accurate. These equations facilitate earlier detection of chronic kidney disease (CKD) and hence appropriate action can be taken to delay its progression. Also appropriate drug dosage in elderly can be given, obviating drug toxicity.

The best known of these equations are the Cockcroft and Gault (CG), the Modification of diet in renal disease study (MDRD) and Chronic Kidney Disease Epidemiology Collaboration study (CKD-EPI). The CG equation is fairly simple, using serum creatinine, ideal body weight, and an adjustment factor for sex. Its main drawbacks are that it was developed to model creatinine clearance, itself an imperfect estimation of GFR and it depends heavily on the accuracy of the value for "lean" body weight used in the equation. ${ }^{1}$

The MDRD and the abbreviated MDRD equation have now largely replaced the CG equation. ${ }^{2}$ Developed using iothalamate GFR measurements, it estimates GFR rather than the less-accurate creatinine clearance (box). Also, it is normalized to a standard body surface area $\left(1.73 \mathrm{~m}^{2}\right)$, obviating the need to determine ideal body weight. The equation has been validated extensively in Caucasian and African American populations between the ages of 18 and 70 with impaired kidney function (eGFR $<60 \mathrm{~mL} / \mathrm{min} / 1.73$ $\mathrm{m}^{2}$ ) and has shown good performance for patients with all common causes of kidney disease. It is superior to traditional methods of approximating GFR like the CG equation and creatinine clearance measured from 24-hour urine collections. The MDRD Study equation is used to assess the burden of chronic kidney disease in epidemiologic studies and public health. The main drawback of the MDRD 
equation is that it tends to underestimate GFR at higher ranges of kidney function, higher than $60 \mathrm{~mL} / \mathrm{min} / 1.73 \mathrm{~m}^{2}$.

The CKD-EPI equation is based on the same four variables as the MDRD Study equation, but uses a different way to model the relationship between estimated GFR and serum creatinine, and a different relationship for age, sex and race (box). The CKD-EPI equation is expected to eventually replace the currently used MDRD equation, as it performs better at higher ranges of GFR. It is as accurate as the MDRD equation at a GFR less than $60 \mathrm{~mL} / \mathrm{min}$ per $1.73 \mathrm{~m}^{2}$ and more accurate at a higher GFR. ${ }^{1}$ The equation was reported to perform better and with less bias than the MDRD Study is the assumption that creatinine production is both stable over time and similar among patients. As a result, these equations should not be used in situations in which renal function is changing rapidly, such as in acute kidney injury. Also, they should be used with caution in patients at the extremes of body mass, since they underestimate GFR in very muscular patients and overestimate GFR in very small patients (malnourished, amputees, vegetarians etc). It is not valid in pregnant women or in children. Furthermore, laboratory differences in creatinine estimations may make significant differences. Probably in the future, other GFR estimating equations may outperform all these equations.

\section{MDRD study equation:}

GFR $=141 \times \min (\mathrm{Scr} / \mathrm{K}, 1)$ y $\times \max (\mathrm{Scr} / \mathrm{K}, 1)-1.209 \times 0.993 \mathrm{age} \times 1.018$ (iffemale) $\times 1.159$ (if black)

$\mathrm{K}=0.7$ if female $\& 0.9$ if male, $\mathrm{y}=0.329$ if female $\& 0.41$ if male, $\mathrm{min}=$ the minimum of $\mathrm{Scr} / \mathrm{K}$ or $1 \mathrm{max}=$ the maximum of $\mathrm{Scr} / \mathrm{K}$ or 1

equation, especially in patients with higher GFR. This results in reduced misclassification of CKD. Also, it was developed with the objective of reporting a specific value even when the estimated GFR is greater than $60 \mathrm{~mL} / \mathrm{min} / 1.73 \mathrm{~m}^{2}$. (In contrast, when laboratories use the MDRD equation, the recommendation is to report any value above this level as "greater than $60 \mathrm{~mL} / \mathrm{min} / 1.73 \mathrm{~m}^{2}$ ).

It is important to remember that eGFR is an estimate of GFR, not the actual GFR. A limitation of all these equations

\section{References}

1. Levey AS, Stevens LA, et al. A new equation to estimate Glomerular Filtration Rate. Ann Intern Med 2009; 150: 604-12.

2. Rule AD, Lieske JC. The estimated glomerular filtration rate as a test for chronic kidney disease: Problems and solutions. Cleveland Clinic Journal of Medicine 2011;78:186-88. 